\title{
EL LIDERAZGO DIRECTIVO EN LA EMPRESA: ¿UNA MERA HABILIDAD O UNA AUTÉNTICA HERRAMIENTA DE DESARROLLO PERSONAL?
}

\section{THE EXECUTIVE LEADERSHIP AT WORK: ¿JUST A MERE SKILL OR A REAL TOOL FOR PERSONAL DEVELOPMENT?}

Pilar Conde Colmenero': Universidad Católica de Murcia (España)

\author{
pconde@ucam.edu
}

\section{RESUMEN}

El liderazgo ha sido entendido tradicionalmente como una habilidad esencial en el ámbito de la gestión empresarial, en especial en lo concerniente a la dirección de los recursos humanos y al fomento de la iniciativa emprendedora. El presente trabajo pretende reflexionar sobre aquellos modelos de liderazgo que pueden constituir, no solo una clave en la gestión del capital humano, sino también una herramienta potenciadora del desarrollo personal de los miembros de la estructura organizativa y del mismo líder emprendedor, siguiendo los postulados de la responsabilidad social corporativa o las últimas iniciativas legales a favor del emprendimiento. Desde esta perspectiva, el liderazgo directivo cobra una nueva dimensión al ser estudiada como fórmula favorecedora del progreso del individuo (conectada a parámetros éticos y de transmisión de valores), que va más allá de las exigencias legales propias de cada ordenamiento jurídico e incluso de la superación de los diversos retos a los que se encuentran expuestos actualmente las organizaciones empresariales. En definitiva, se trata de asumir unas prerrogativas éticas a la hora de liderar a las personas; cuestión esta que parece indispensable en las modernas sociedades azotadas por crisis financieras que han evidenciado los puntos débiles de un sistema de mercado marcado por conductas corporativas poco honestas.

PALABRAS CLAVE: Liderazgo directivo - gestión de recursos humanos responsabilidad social corporativa - desarrollo personal - modelos directivos exigencias éticas - transmisión de valores.

\footnotetext{
${ }^{1}$ Pilar Conde Colmenero: Universidad Católica de Murcia, Departamento de Ciencias Sociales, Jurídicas y de la Empresa. Murcia .España. Formación docente Evaluación Positiva del Ministerio de Educación, Cultura y Deporte y de la ANECA para las figuras de Profesor Contratado Doctor, Profesor de Universidad Privada y Profesor Ayudante Doctor (2010) Licenciada en Derecho (UJA, 1994) Licenciada en Filosofía. UGRA, 1988) Máster MBA (ICADE, 1990) Certificado de Aptitud Pedagógica -CAP (UGRA, 1988) Doctora en Derecho del Trabajo y de la Seguridad Social (URJC, 2007)
} 


\section{ABSTRACT}

Leadership has been traditionally seen as an essential Skull in the field of Business administration, especially in the areas concerning the human resources Management and the enterprising initiative. The aim of this present project is to think about those leadership models that can constitute, not only the key when dealing with employees but also a enhancing tool of the personal and human development of the staff members and the enterprising leader himself - following the postulates of corporate social responsibility or the last legal initiatives proposed in favor of enterprising. From this viewpoint, this executive leadership gains a new dimension being studied like a formula which enables the progress of the individual (linked to ethical and valuetransmitting parameters), which goes further than the legal requirements typical of every legislation and also even further than the achievement of diverse challenges which companies are exposed to nowadays. Summarizing, it deals with assuming some ethical prerogatives when leading with people - an issue that seems essential in modern societies hit by financial crisis that have made clear which are the weak points of market-ruled system affected by not very honest corporative behaviors.

\section{KEY WORDS: Executive leadership - Human Resources Management - Corporate Social Responsability - Personal Development - Executive Models - Ethical Requirements - Value-Transmitting.}

\section{Cómo citar el artículo:}

Pilar Conde Colmenero. (2013) EL LIDERAZGO DIRECTIVO EN LA EMPRESA: ¿UNA MERA HABILIDAD O UNA AUTÉNTICA HERRAMIENTA DE DESARROLLO PERSONAL?. Revista de Ciencias de la Comunicación e Información. (Diciembre 2013). Año XXVII (30), 36-49 ISSN: 0213-070-X. Recuperado de: http://www.revistaccinformacion.net/ARTICULOS/2013\%20e-j\%20Bea/2013\%20e\%20El\%20liderazgo\%20directivo\%20en\%20la\%20empresa.\%20Pilar\%20Conde\%20(U CAM).pdf

\section{INTRODUCCIÓN²}

El liderazgo ha sido entendido tradicionalmente como una habilidad esencial en el ámbito de la gestión empresarial, en especial en lo concerniente a la dirección del personal y al fomento de la iniciativa emprendedora. Significa, en todo caso, un acercamiento al mundo de la dirección y dirección de empresas desde una óptica más humana.

\footnotetext{
${ }^{2}$ Este artículo es resultado del proyecto de investigación 18889/OC/13 financiado por la Fundación Séneca- Agencia de ciencia y Tecnología de la Región de Murcia en el marco del III PCTRM 20112014.
} 
Desde estas líneas se pretende reflexionar sobre aquellos modelos de liderazgo que pueden constituir, no solo una clave en la gestión de los recursos humanos (que contribuya de manera eficaz al éxito de la actividad económica de la empresa), sino también una herramienta potenciadora del desarrollo personal y humano de los miembros de la estructura organizativa, siguiendo los postulados de la responsabilidad social corporativa.

Desde esta perspectiva, el liderazgo directivo cobra una nueva dimensión al ser estudiada como fórmula favorecedora del progreso del individuo (conectada a parámetros éticos y de transmisión de valores), que va más allá de las exigencias legales propias de cada ordenamiento jurídico e incluso de la superación de los diversos retos a los que se exponen actualmente las organizaciones empresariales. En definitiva, se trata de asumir unas prerrogativas éticas a la hora de liderar a las personas; cuestión esta que parece indispensable en las modernas sociedades azotadas por crisis financieras que han evidenciado los puntos débiles de un sistema de mercado marcado por conductas corporativas de gestión fraudulenta 0 , como mínimo, poco honestas.

\section{OBJETIVOS}

El objetivo último del presente estudio consiste en argumentar la solvencia, necesidad y utilidad de aquellos modelos de liderazgo que apuestan por el desarrollo personal de los empleados que conforman la organización empresarial (o del mismo líder emprendedor), así como por una transmisión de valores y un respeto a los derechos humanos y a la dignidad personal que redunde en una gestión de negocios comprometida con la sociedad y los propios empleados.

\section{METODOLOGÍA}

La metodología empleada en el presente trabajo, al tomar éste forma de ensayo, no es la propia de una investigación empírica sino que se encuentra más cercana a la revisión bibliográfica. Partiendo de la hipótesis de que actualmente, en la delicada situación de crisis económico-financiera que se vive, existe una necesidad de retomar fórmulas de gestión empresarial con compromiso ético, se pretende comprobar la posible conexión entre aquellos modelos de liderazgo de los recursos humanos en la empresa que apuestan por fórmulas de desarrollo personal y la superación de las deficiencias de formación en valores de los modernos directivos, detectadas en el panorama mercantil de la última década; todo ello basado, tanto en los modernos postulados de la responsabilidad social corporativa, como en las propuestas doctrinales que abogan por una organización empresarial que se debe a sus empleados, a sus socios, o las propias comunidades donde se incardinan y para las que realizan su actividad económica.

Para ello, es imprescindible abordar la aplicación de las teorías sobre la responsabilidad social corporativa (manejando la literatura científica de sus más emblemáticos representantes) y los modernos modelos de liderazgo directivo, tras chequear el panorama de la gestión empresarial llevada a cabo durante la época de crisis. 


\section{RESULTADOS}

\subsection{El liderazgo directivo y las decisiones empresariales en el actual escenario de crisis económica}

Desde que a la altura del año 2007 comenzase en EEUU, propagándose rápidamente en cadena a Europa, el declive de algunas grandes entidades financieras (debido en gran medida a la comercialización de los llamados créditos subprime), y se provocase la lamentable crisis global en la que está inmersa España y otros muchos países europeos, ha cundido un sentimiento social de reproche por la gestión poco decorosa llevada a cabo desde determinadas entidades -bancos mayoritariamente, aunque también empresas de otros sectores de la economía (De la Torre-Olid, 2013).

Estos acontecimientos se unen a una preexistente demanda social que exigía a las corporaciones mercantiles un mayor compromiso con la comunidad y los derechos humanos. Cuestión esta que encuentra su fundamento en la reciente preocupación de los países occidentales por la responsabilidad de las empresas respecto de las consecuencias sociales, económicas y medioambientales de su actividad a raíz de circunstancias como la globalización económica, la degradación del medioambiente, la intolerancia frente a prácticas de explotación laboral o la concienciación de la necesidad de transparencia en la gestión empresarial (Conde-Colmenero, 2009).

La presente situación de crisis no ha hecho sino acentuar el protagonismo de estas inquietudes y el surgimiento particular de propuestas legales y de soft law (Derecho blando) que ofrecen fórmulas, pautas y herramientas eficaces para solventar las singulares dificultades económicas de comienzos del siglo XXI y, a su vez, cumplir con las nuevas exigencias sociales.

De otro lado, lo que comenzó siendo un problema en principio circunscrito al plano financiero derivó, con el transcurrir del tiempo, en un grave crecimiento del desempleo motivado por la destrucción exponencial de tejido empresarial que ha provocó el fuerte declive económico. Desempleo del que no se han visto libres ni siquiera grandes potencias como Estados Unidos o Alemania, si bien sus tasas de desocupación (del $8 \%$ aproximadamente) les colocan en una mejor posición en comparación con países como España que superan el $25 \%$ de índice de desempleo (pese a la contundencia del artículo 40 de la Constitución de 1978 que propugna que los poderes públicos realizarán una política orientada al pleno empleo). En efecto, según datos oficiales en nuestro país la tasa total de desempleo, a fecha de enero de 2013 , se encuentra en un $25,8 \%$ (afectando más crudamente a determinados colectivos como las mujeres, con un $26,8 \%$, y a los menores de 25 años, con un $54,6 \%)$.

En estos delicados momentos económicos, emergen con fuerza, desde las mismas autoridades, iniciativas que persiguen fomentar el emprendimiento y otorgar la oportuna importancia al liderazgo emprendedor, además de servir de recetas efectivas contra el paro y el deterioro productivo. Estas decisiones legislativas se amparan, como no puede ser de otra manera, en la importancia que la propia Carta Magna otorga a la participación ciudadana afirmando con rotundidad que a los 
poderes públicos les corresponde facilitar la participación de todos los ciudadanos en la vida política, económica, cultural y social (art. 9 Constitución Española) y, a su vez proclamando el derecho de los ciudadanos a la participación directa en los asuntos públicos (art. $23 \mathrm{CE}$ ).

En íntima relación con lo apuntado se encuentra la emergencia de la Ética Empresarial (incardinada en la rama de la Filosofía social y Ética de las organizaciones), no sólo como disciplina científica que ha tenido una reciente ascensión, sino como corriente de pensamiento en alza y, en definitiva, como auténtico clamor popular de los ciudadanos ante la actuación de entidades financieras y poderes públicos en los tiempos previos a la crisis y posteriores a la misma. En efecto, en los últimos años se viene constatando una desatada tendencia a vincular la palabra "ética" y la palabra "empresa", probablemente debido a las desafortunadas decisiones de algunas firmas comerciales y a una mayor sensibilidad social respecto del ejercicio de la actividad mercantil y sus efectos sobre los propios empleados y la comunidad en general. Más que a una "moda" esta vinculación ética-empresa responde a una reacción social crítica ante bastantes actuaciones que han tenido lugar últimamente en toda clase de organizaciones, tanto empresas privadas como Administraciones Públicas (Lozano, 1999), algunas de las cuales aluden directamente a temas de gestión de personal y liderazgo directivo.

A resultas de ello, las escuelas de negocios y las instituciones universitarias, sobre las que recae por excelencia el peso de la formación superior y de especialización, han retomado con fuerza sus reflexiones sobre el compromiso ético de las empresas en la toma de decisiones, la dirección de los recursos humanos y el diseño de políticas comerciales, poniendo especial acento en cuestiones deontológicas, revisando sus pronunciamientos sobre gestión empresarial transparente y ética, y reformulando herramientas de trabajo encaminadas a la mejora de la misma. Obviamente estas cuestiones venían ocupando ya un lugar en los planes de estudios de las entidades dedicadas ex profeso a la formación especializada de directores ejecutivos, sin embargo ahora se comprueba que deben tener un sitio preeminente y deben enfocarse desde nuevas ópticas puesto que la experiencia económica vivida y los factores que la han provocado hacen necesaria su revisión.

En definitiva, importantes especialistas científicos y autoridades morales advierten que la crisis económica no se puede desligar de la profunda crisis de valores que padecen las sociedades postmodernas de nuestro tiempo y que, como no, afecta también a la dirección empresarial (Dolan y García, 2001).

En este contexto, meditar sobre el ejercicio del liderazgo, y más en concreto sobre el concepto de liderazgo socialmente responsable, constituye un tema de suma actualidad y de importancia capital, sobre todo a la luz de las últimas pésimas experiencias económicas sufridas en los países industrializados y de los desaciertos producidos en el campo de la dirección empresarial.

\subsection{Definición, contenidos y caracteres del liderazgo}


Es de todos conocido que, en el ámbito empresarial, liderar implica motivar, comunicar, persuadir e influir en los miembros de la organización de manera que se consigan obtener las metas comunes perseguidas en la actividad económica llevada a cabo por cada corporación (García-García y Pomares- Barriocanal, 2008; PeñaAcuña, 2005).

Descendiendo a detallar las competencias y conductas señaladas tradicionalmente por la literatura especializada (Gómez-Megía, Balkin, y Cardy, 2006; Leal-Millán, Alfaro de Prado-Sagrera, Rodríguez-Felix y Román-Onsalo 1999), el buen líder debe saber identificar y satisfacer las necesidades de las personas a su cargo (entre ellas las de desarrollo personal y aprendizaje), exigir responsabilidad a los que le rodean, ser ejemplo de honradez e irradiar confianza y seguridad, ser comprometido con los objetivos de la empresa (en su sentido más extenso, incluyendo los objetivos sociales y medioambientales), tratar de manera considerada y con dignidad a los empleados, apreciándolos sinceramente en el plano personal y profesional, entre otras cosas (García-García y Pomares-Barriocanal, 2008). En definitiva, a juicio de la doctrina más solvente (Chiavenato, 2001), el líder directivo ejemplarizante es aquel que sabe crear un clima organizacional y un ambiente laboral óptimo, por tanto, un contexto favorecedor del progreso y mejora de los individuos, tanto en el plano personal como colectivo.

Bajo ese prisma, liderazgo directivo y desarrollo personal van de la mano, es decir se encuentran íntimamente relacionados puesto que el buen líder es aquel que, bajo las circunstancias económico-empresariales singulares de nuestro tiempo, ejerce de guía del grupo fomentando, por ejemplo, el aprendizaje permanente de los empleados, la responsabilización de los trabajadores, el impulso de las capacidades individuales, la comunicación franca, la conciliación de la vida familiar y profesional, etc.

Esas cualidades y actitudes descritas conducen entonces, de forma natural, a la definición de líder socialmente responsable; es decir, si se examinan los caracteres definidores del liderazgo directivo automáticamente se detecta el paralelismo con las exigencias propias del liderazgo responsable, cercano al "jefe ideal" (Freemantle, 1993), que propicia, siempre, un desarrollo humano de los empleados y que es coherente con un trato respetuoso y digno a los trabajadores y a quienes podrán ser seleccionados para incorporarse en el futuro a la empresa. En radical oposición a esa concepción del líder socialmente responsable, cuya noción se desarrolla extensamente a continuación, se sitúa el líder directivo, no ya incompetente (Carreño, 1996), sino tóxico u hostigador, que se aleja sustancialmente de parámetros éticos y descuida de manera preocupante el lado humano de la dirección de las personas en la organización empresarial (Odiorne, 1989). También llegado el momento, se tratará con amplitud el concepto de líder directivo acosador ahora anunciado.

\subsection{Ejercicio comprometido del liderazgo y responsabilidad social corporativa (RSC)}

El ejercicio del liderazgo no supone per se una utilización responsable de la habilidad de influir en los demás para acometer con entusiasmo objetivos comunes. 
Sólo el liderazgo responsable, fiel a unos valores, a la propia naturaleza humana en su dignidad y a unos fines honestamente consecuentes (más allá de las mismas exigencias legales) es el auténticamente defendible y, por ende, admisible en una sociedad que pugna por el retorno a unos deteriorados principios de gestión ética. Por tanto, el liderazgo directivo debe ser utilizado, lógicamente, para cubrir metas económicas, mercantiles y de búsqueda legítima de beneficios, aunque sin alejarse del cumplimiento de pautas éticas que lo desnaturalizarían y lo harían caer en un liderazgo pernicioso para la sociedad y para la misma empresa que lo practica (a través de sus directivos). Es más, el uso del liderazgo en pro del rendimiento económico debe ser compatible con el ejercicio responsable del mismo, o dicho en otros términos, ambos deben poder coincidir.

En esa línea, los poderes públicos (nacionales e internacionales) y las mismas compañías comienzan a comprender y vislumbrar, desde hace unos años, la necesidad de proclamar que las empresas han de actuar de manera responsable frente a sus empleados, clientes, proveedores, accionistas, consumidores y usuarios, Administraciones públicas, etc. (los denominados stakeholders o sujetos afectados por la actividad de las entidades mercantiles), so pena de que la imagen de su actividad comercial se vea empañada y el público (la sociedad) penalice sus actividades inapropiadas dejando de consumir sus productos y servicios. Se trata pues de ahondar en el problema de la eficiencia y la eficacia organizacional y las críticas formuladas a su evaluación cuantitativa, que sólo incluye los aspectos financieros y contables perdiendo de vista los aspectos humanos, reales y potenciales (Chiavenato, 1996).

A esta toma de conciencia se debe el auge obtenido por diversas iniciativas europeas y nacionales en pro de la denominada "responsabilidad social corporativa" o "responsabilidad social empresarial" (en adelante RSC o RSE, respectivamente), entendiendo que, en el marco de la gestión empresarial y en particular en lo referente a la dirección de los recursos humanos, actuar de manera responsable incluye liderar de manera responsable. En el concepto de responsabilidad social corporativa quedan comprendidas, entre otras, las cuestiones relativas a la dirección de los empleados $\mathrm{y}$, por tanto, todo lo concerniente a un liderazgo comprometido.

Caminando en este sentido, el Libro Verde, de la Comisión Europea, para "Fomentar un marco de Responsabilidad Social de las Empresas", presentado en Bruselas el 18 de julio de 2001, después de definir las RSC como la integración voluntaria, por parte de las empresas, de las preocupaciones sociales y medioambientales en sus operaciones comerciales y sus relaciones con sus interlocutores, manifiesta que las prácticas responsables en lo social afectan en primer lugar a los trabajadores y se refieren a cuestiones como la inversión en recursos humanos, la salud y la seguridad, y la gestión del cambio.

El noble objetivo que persigue la RSE es conseguir una empresa mejor para un mundo mejor, es decir se trata, en definitiva, del viejo postulado de que la empresa devuelva a la sociedad la riqueza que ésta le da (Conde-Colmenero, 2008). Para ello, es necesario aceptar que la actuación de la empresa no puede ya quedar dirigida en exclusiva a la mera obtención del máximo beneficio económico posible y 
al cumplimiento mínimo y rutinario de las exigencias legales, sino que han de valorarse los efectos que la actividad de la organización productiva puede generar para todos los sujetos potencialmente afectados y plantearse seriamente ir más allá de los meros requisitos jurídicos, ya que de no ser así debe aceptar la idea de pagar una "factura social" y afrontar el reproche de la comunidad.

Previamente, es necesario aclarar que la RSC posee con carácter general dos vertientes, una interna y otra externa. Este doble nivel de proyección de la responsabilidad social de las corporaciones puede también mantenerse cuando se trata de liderazgo directivo. Así:

- Ad intra, hacia los propios empleados, el comportamiento con ellos y el trato acorde a su dignidad: en esta vertiente se localizan las cuestiones sobre dirección y gestión del capital humano en la empresa, por tanto también el ejercicio del liderazgo como habilidad directiva fundamental capaz de generar un marco favorecedor del desarrollo personal. En este mismo plano, se incardina todo lo relativo a las políticas empresariales de relaciones laborales y las decisiones sobre seguridad y salud en el trabajo; ambas temáticas, a su vez, conectan de modo natural con el ejercicio del liderazgo responsable

- Ad extra, hacia la sociedad en su conjunto y hacia la comunidad local donde opera la empresa en particular fundamentalmente a través de la relación con sus clientes y futuros empleados: en esta vertiente también ocupa un lugar principal la dirección de los recursos humanos, entendida ésta en un sentido amplio que incluye la necesidad de la empresa de atraer a los trabajadores más cualificados mediante una correcta evaluación de las capacidades personales que requiere la organización y de la fijación del modelo de trabajo colectivo y liderazgo que se desee establecer en la corporación, así como a través de una acertada selección del personal (sin prácticas discriminatorias, comprometida con las necesidades sociales, etc), de una contratación ajustada a las exigencias legales y a los derechos de los trabajadores (con una actitud intolerante respecto de la explotación laboral o el fraude en la contratación temporal, etc) y de la creación y redistribución de empleo, entre otras.

Cuando este importante documento comunitario especifica los desafíos más importantes a los que se enfrentan las empresas actualmente en materia de gestión y políticas de recursos humanos, así como las medidas pertinentes para superar estos retos hace referencia expresa al fomento del aprendizaje permanente, de la responsabilización de los trabajadores y de la conciliación entre trabajo, familia y ocio.

De entre las medidas apuntadas, suscita especial interés para las instancias europeas el ámbito del aprendizaje permanente, circunscrito en particular a la dirección de los empleados ejercida principalmente a través de sus directivos. A juicio del Libro Verde, las empresas deben desempeñar un papel fundamental respecto del aprendizaje permanente, en varios niveles entre los que destacan: 
- Contribuir a definir mejor las necesidades de formación mediante una asociación estrecha con los agentes locales que diseñan los programas de educación y formación.

- Fomentar el paso de los jóvenes de la vida escolar y universitaria a la vida laboral, valorando el aprendizaje y facilitando por ejemplo el acceso a puestos de aprendizaje y en prácticas.

- Crear un entorno que estimule el aprendizaje permanente de todos los trabajadores, en particular de los trabajadores con un menor nivel educativo y de cualificación, así como de los trabajadores de mayor edad.

Es fundamental recordar que este apoyo al fomento del aprendizaje por parte de los líderes directivos implica sencillamente ser consecuente con las exigencias legales, e incluso ir más allá de las mismas conforme a los postulados de la RSC, ya que entre los derechos laborales básicos de los trabajadores se encuentra el derecho a la promoción y formación profesional (tal como dispone, dentro del ordenamiento jurídico español, el artículo 4.2 letra b del Estatuto de los Trabajadores).

En esa línea argumentativa interesa recordar que, en paralelo a la dimensión (interna y externa) de la dirección de los recursos humanos que posee la RSE corre la dimensión jurídico-laboral, cuyos contenidos principales conectan, como se va comprobando, directamente con el liderazgo socialmente responsable (y que deben ser atendidos por quienes lo ejercen) al proporcionar relevancia, por ejemplo, al respeto a los derechos humanos y laborales fundamentales (con especial referencia a la lucha contra la explotación laboral), la inquietud por consolidar un empleo de calidad y por la mejora constante de las condiciones sociolaborales de los trabajadores en la empresa -como la igualdad de oportunidades y la no discriminación, el favorecimiento de la formación y promoción profesional, las buenas prácticas en prevención de riesgos laborales, la integración en la empresa de personas con dificultades de inserción laboral y discapacidad, la conciliación de la vida laboral y familiar, etc - (Conde-Colmenero, 2009), así como al diseño, cada vez más intenso y amplio, de beneficios y acciones sociales.

Concluyendo, en materia de RSC ligada al liderazgo directivo, entre los temas que se consideran prioritarios abordar desde Europa se encuentran el impulso del aprendizaje a lo largo de la vida laboral y de la ética como instrumento adicional que sirva a la gestión del cambio industrial y a las reestructuraciones empresariales, amén del resto de facetas directivas e iuslaboralistas mencionadas que se encuentran directamente vinculadas con ellas. En consecuencia, los contenidos de gestión de los recursos humanos y sociolaborales siguen siendo objetivo preferente en la composición de las modernas tendencias sobre responsabilidad social corporativa, lo que sustenta la idea de apostar por un liderazgo socialmente responsable y propiciador a ultranza del desarrollo personal.

Es más, las últimas tendencias europeas, y también internacionales, en materia de RSE apuntan, no tanto por avanzar en la teorización sobre el necesario "balance social" de las empresas (que es de todo punto indiscutible y se encuentra en una etapa de madurez), sino por cuestiones más prácticas y reales que se concreten en 
propuestas de futuro como mejorar el grado de integración de las políticas de responsabilidad social, incentivar la viabilidad práctica y efectividad de las mismas, el perfeccionamiento de los instrumentos específicos de la RSC (tales como la llamada "auditoria social", la certificación vía estándar AS: 8000 , las memorias de sostenibilidad o los códigos de conducta, etc), etc. Ahí tienen cabida, sin duda, todas las propuestas que el presente artículo plantea en pro de un líder directivo socialmente responsable que se propugne favorecedor de su propio desarrollo personal y el de su equipo.

\subsection{El líder acosador: contrapunto del líder socialmente responsable}

En contraposición al liderazgo responsable, cuyas características y fundamentación se han expuesto anteriormente, se encuentra el caso del líder acosador, es decir el supuesto de quien abusa del poder de dirección y dispensa un trato denigrante a sus empleados, cuestión esta que afecta particularmente a la gestión del personal y que en las últimas décadas se ha convertido en un problema alarmante por su grado de expansión en las empresas, según reflejan los informes especializados y estadísticas más actualizadas (como la Encuesta Europea de Condiciones de Trabajo para 2013).

A fin de comprender claramente lo que debe ser un buen líder (un líder responsable que permite el desarrollo personal de su personal y el suyo propio, según el prototipo que se viene defendiendo desde este estudio) es oportuno examinar a fondo las conductas directivas que se incardinan en el lado opuesto: las conductas de hostigamiento o el denominado acoso, en su modalidad de "acoso descendente" -en terminología de los psicólogos del trabajo y los iuslaboralistas -, es decir el trato denigrante que puede realizar un superior sobre sus subordinados.

Con carácter previo y desde una perspectiva jurídica, ya que en nuestro país las conductas acosadoras en el ámbito laboral están especialmente sancionadas por el Derecho desde 2007, conviene explicar que la titularidad del poder de dirección corresponde al empresario, si bien el ejercicio de dicho poder se delega comúnmente en directores profesionales, que a su vez suelen delegar parcelas de su poder general en otros mandos inferiores. Por tanto el ejercicio abusivo y hostigador del liderazgo directivo puede efectuarse por el mismo empresario y/o a través de sus directivos.

Pues bien, el contrapunto del líder ideal, del líder que contribuye a su propio desarrollo personal y al de aquellos que lo rodean, lo constituye el líder acosador, es decir aquel que con su manera de dirigir vulnera la dignidad de sus empleados y realiza un ejercicio abusivo de su poder de dirección mediante un liderazgo deshonesto, falto de valores y de consideración hacia los trabajadores y sus derechos como individuos.

Ante todo conviene subrayar que las conductas acosadoras en el trabajo son consideradas como ilícitos graves, de relevancia constitucional, ya que conllevan una vulneración de la dignidad de los empleados que las padecen y suponen un atentado a la integridad física y moral de la persona, por lo que vulneran derechos fundamentales constitucionalmente reconocidos (artículo 10 y 15 principalmente). 
Fue el profesor sueco Leymann (1996) quien de forma precursora y desde una perspectiva psicosocial inició los estudios científicos sobre el hostigamiento laboral y con sus investigaciones hizo recapacitar a los especialistas sobre la expansión preocupante de estas actitudes entre los directivos y líderes empresariales de las compañías europeas.

Las conductas acosadoras empleadas por los jefes y directivos contravienen de manera flagrante la concepción de la empresa como entramado de relaciones interpersonales jerarquizadas que deben desarrollarse de manera sana y humanizada, generando un ambiente de trabajo saludable, para no derivar en actitudes nocivas y perniciosas para la salud de los trabajadores que redunden en un deterioro personal (físico o psíquico), familiar y profesional grave.

Desde los años 90 y merced a los numerosos estudios realizados en el contexto europeo se ha generado una concienciación de los poderes públicos y las empresas respecto de la emergencia del acoso laboral creándose instrumentos jurídicos para hacerle frente de forma efectiva. Baste señalar a modo de ejemplo, la firma del Acuerdo Marco Europeo sobre acoso y violencia en el trabajo (2007) por parte de las principales patronales y sindicatos con representación a nivel comunitario, que ocupa un lugar preeminente entre los avances operados en la materia.

Las investigaciones de psicólogos y sociólogos del trabajo sobre el perfil del acosador ayudan a identificar éste con el perfil del líder que no es responsable socialmente y que, antes que fomentar el desarrollo personal de los empleados, ejerce un influjo altamente negativo y dañino para los individuos que le rodean. Así, desde una óptica psicológica, social, ética y conductual, el individuo con tendencias hacia la práctica del hostigamiento suele ser descrito como alguien (Hirigoyen, 2001 y Piñuel- Zabala, 2004):

- autoritario en exceso.

- inseguro de sí mismo, por lo que no le gusta rodearse de personas competentes que le puedan hacer sombra profesionalmente.

- mediocre en lo personal y en lo profesional, desarrollando un recelo extremo respecto de los méritos de sus propios subordinados y un desasosiego por que lo superen o eclipsen.

- altamente controlador, de manera que impide el trabajo autónomo de sus empleados, obstaculiza la delegación de funciones y no favorece la formación y promoción de quienes le rodean.

- narcisista, con propensión a la megalomanía.

- poco dado a compartir méritos y recompensas con los miembros de su propio equipo.

- falto de sentimiento de responsabilidad por sus actos. 
Tal como se desprende de esta descripción, y según se viene anunciando, el líder socialmente responsable, el que ejerce una habilidad directiva tan importante de modo ejemplar permitiendo el desarrollo personal de su personal (y el suyo propio), es el más alejado del líder acosador.

\subsection{La formación en liderazgo: una cuestión de educación temprana}

La creación de programas formales y planificados orientados hacia el desarrollo del talento directivo y el adiestramiento de líderes tiene una larga trayectoria aunque, desde una perspectiva histórica, es un hecho relativamente reciente que los especialistas en dirección empresarial sitúan fundamentalmente tras la Segunda Guerra Mundial (Odiorne, G.S., 1989), cuando se dieron una serie de circunstancias, como la escasez de directivos, por las cuales comenzaron a proliferar los planes de desarrollo de ejecutivos universitarios que no ha ido sino creciendo vertiginosamente en la últimas décadas.

La novedad en nuestro país reside en que muy recientemente los legisladores han decidido promover la formación de los emprendedores y líderes a temprana edad, entre otros motivos para propulsar el imprescindible espíritu del emprendimiento tras la recesión económica acontecida y la alarmante destrucción de tejido productivo.

Comienza a ser unánime la idea de que la formación de los futuros líderes se revela imprescindible y debe ser acometida en fases educativas tempranas puesto que el impulso de ciertas competencias y aptitudes propias de quienes dirigirán a personas, en su propia empresa o en el marco de una corporación, no está reservado sólo a la enseñanza superior universitaria o de especialización sino que debe estar implementado en los niveles formativos básicos. De este modo se consigue, de un lado impregnar a niños y jóvenes del espíritu inherente al liderazgo y al emprendimiento, y de otro instruir al alumno desde muy pronto y de manera efectiva en las funciones, contenidos y características que los definen.

No en vano la flamante Ley 14/2013, de 27 de septiembre, de apoyo a los emprendedores y su internacionalización aborda, de manera novedosa en su articulo 4, la cuestión del emprendimiento en la enseñanza primaria y secundaria exigiendo que los currículos de los profesores de estos niveles incorporen objetivos orientados al desarrollo y afianzamiento del espíritu emprendedor y de la ética empresarial. El exponente máximo del líder es aquel que es líder de sí mismo y de su propia iniciativa emprendedora y empresarial, por lo que formar y fomentar entre el alumnado de primaria y secundaria medidas y actitudes como la creatividad, la confianza en uno mismo o la ética empresarial, como lo hace esta normativa, son el germen del liderazgo bien entendido.

Las previsiones de esta flamante Ley tienen su raíz en lo dispuesto por la última reforma normativa sobre educación que ha visto la luz en nuestro país, la Ley Orgánica 8/2013, de 9 de diciembre, para la mejora de la calidad educativa (identificada bajo las siglas LOMCE) que pone especial énfasis, en su Exposición de Motivos, en que el sistema debe ser capaz de encauzar a los estudiantes hacia rutas que estimulen el espíritu emprendedor (capacidad de liderar organizaciones empresariales) dando la posibilidad de elegir las mejores opciones de desarrollo personal y profesional. Esto no significa sino que desde el sistema de enseñanza se 
debe capacitar a los alumnos en la habilidad de liderar su propia empresa como fórmula de desarrollo de capacidades personales y profesionales.

Por tanto, la apuesta actual de los poderes públicos en materia educativa se inclina por el fomento a ultranza del liderazgo emprendedor y directivo desde los niveles más básicos de la enseñanza, dando el relevo posteriormente, en edades menos precoces, a las instituciones universitarias y escuelas de negocios que habitualmente han venido preparando a los ejecutivos en programas de especialización.

\section{CONCLUSIONES Y DISCUSIÓN}

PRIMERA: El escenario actual de crisis ha colocado en primera línea la reflexión sobre la ética empresarial y el ejercicio responsable del poder directivo. De la mano de estas cuestiones, nace el análisis del liderazgo directivo como herramienta de desarrollo personal que propicie una vuelta a la, tal vez relegada, dirección por valores.

SEGUNDA: Una vez examinados los caracteres propios del liderazgo en la empresa queda constatado que el ejercicio adecuado de esta habilidad directiva se encuentra íntimamente vinculado al desarrollo humano y personal de los empleados, e incluso del propio líder, según las últimas tendencias sobre responsabilidad social corporativa aplicadas al ámbito de la gestión y dirección de los recursos humanos. Es factible, por tanto, alcanzar la convicción de que el auténtico líder, sobre todo en el presente panorama económico y social, es aquel que dirige el capital humano de la empresa bajo los parámetros de la RSC, haciendo una firme apuesta por el aprendizaje continuado y la ética en la gestión.

TERCERA: El líder socialmente responsable es el paradigma del liderazgo, entendido este como una auténtica herramienta de desarrollo personal en la empresa, sobre todo porque favorece la formación profesional y estimula el aprendizaje permanente de todos los trabajadores, en particular de los trabajadores con un menor nivel educativo y de cualificación, así como de los trabajadores de mayor edad.

CUARTA: En el polo opuesto al líder socialmente responsable se encuentra el líder acosador, es decir aquel que ejerce su influencia sobre los empleados sin atender a la dignidad personal de los mismos y que ataca sus derechos fundamentales y laborales básicos, mediante conductas hostigadoras que obstaculizan su formación y promoción, con un trato despectivo, etc. Tales conductas de acoso en el trabajo están en la actualidad sancionadas por nuestro ordenamiento jurídico, tanto en el plano laboral como en el plano penal, a semejanza de lo legislado en los países europeos de nuestro entorno más cercano y en el resto de naciones industrializadas situadas a la vanguardia de los derechos humanos y de los trabajadores.

QUINTA: La formación en liderazgo, desde criterios éticos y de responsabilidad social, debe iniciarse en edades tempranas. Solo así se garantiza el futuro surgimiento de líderes emprendedores y directivos consecuentes con las exigencias sociales y morales del mundo actual. Es encomiable por tanto que el legislador español venga haciendo una apuesta decidida por introducir la formación de los 
líderes emprendedores en nuestro sistema educativo (particularmente con su última normativa reformadora del sector - la Ley Orgánica 8/2013, para la mejora de la calidad educativa, LOMCE - y de apoyo al emprendimiento).

\section{REFERENCIAS}

Carreño, P. (1996). Pilato o de la Incompetencia Directiva, Madrid: Editorial AC.

Chiavenato, I. (2001). Administración de Recursos Humanos. Santafé de Bogotá: McGraw-Hill.

Conde-Colmenero, P. (2008). El acoso laboral en el empleo público. Granada: Comares.

Conde-Colmenero, P. (2009) "La responsabilidad social de la empresa desde el Derecho Social Comunitario", Capítulo de la obra colectiva Cuestiones actuales sobre Derecho Social Comunitario, VV. AA. (Areta-Martínez, M. y Sempere-Navarro, A.V. -Codtores.-), Murcia: Laborum, p. 619-631.

De la Torre-Olid, F. (2013): "Actualidad de los cuasi contratos. Revitalización del art. 1891 CC para la tutela de los consumidores-inversores en el sector financiero (un estudio necesario en tiempos de crisis)", Revista Crítica de Derecho Inmobiliario, ํㅜ 739, p. 2951-2982.

Dolan, S.L. y García, S. (2001). La Dirección por Valores. Madrid: McGraw-Hill.

Freemantle, D. (1993). Jefes Increibles. Santafé de Bogotá: McGraw-Hill.

García-García, R. y Pomares-Barriocanal, J.F. (2008): Habilidades Profesionales. Murcia: DON Consultores.

Gómez-Megía, L.R.; Balkin, D.B. y Cardy, R.L. (2006). Dirección y Gestión de Recursos Humanos. Madrid: Prentice Hall.

Hirigoyen, M.F. (2001): El acoso moral en el trabajo, distinguir lo verdadero de lo falso. Barcelona: Paidós.

Leal-Millán, A.; Alfaro de Prado-Sagrera, A.; Rodríguez-Felix, F. y Román-Onsalo, M. (1999). El factor humano en las relaciones laborales. Madrid: Pirámide.

Leymann, H. (1996). Mobbing: la persécution au trabail. Paris: Seuil.

Lozano, J.M. (1999). Ética y Empresa. Madrid: Editorial Trotta.

Odiorne, G.S. (1989). El lado humano de la dirección. Madrid: Ediciones Díaz de Santos.

Peña-Acuña, B. (2005). La dirección de comunicación y las habilidades directivas. Revista de la Seeci, Año VIII, nº 12, noviembre, p. 36-54.

Piñuel-Zabala, I. (2004): Neomanagement: Jefes tóxicos y sus víctimas. Madrid: Aguilar. 